\title{
Sexual selection against deleterious mutations via variable male search \\ success
}

\author{
Kelsie MacLellan ${ }^{1}$, Michael C. Whitlock ${ }^{2}$ \\ and Howard D. Rundle ${ }^{1, *}$ \\ ${ }^{1}$ Department of Biology and Centre for Advanced Research in \\ Environmental Genomics, University of Ottawa, Ottawa, \\ Ontario, Canada K1N 6N5 \\ ${ }^{2}$ Department of Zoology, University of British Columbia, Vancouver, \\ British Columbia, Canada V6T $1 Z 4$ \\ *Author for correspondence (hrundle@uottawa.ca).
}

In many species, successful mating requires the initial step of actively searching for and locating a female. The overall health or condition of a male is likely to affect their ability to do this, making search effort a potentially important component of sexual fitness that may have important consequences for population mean fitness. We investigated the potential population genetic consequences of search effort using 10 populations of Drosophila melanogaster, each fixed for a different recessive mutation with a visible phenotypic effect. Mate choice trials were conducted in arenas of varying size, requiring different levels of search ability. Sexual selection against mutant males was stronger when increased search effort was included than when it was excluded. Varying abilities to find mates can substantially increase the strength of selection against deleterious alleles.

Keywords: Drosophila melanogaster; mate search; mutation load; reproductive success; scramble competition; search effort

\section{INTRODUCTION}

Sexual selection is a process that occurs when a non-random relationship exists between trait variation and an individual's reproductive success. In most species males compete, either directly or indirectly (Murphy 1998), for access to females and females discriminate among potential males via pre- and/or postcopulatory processes. Successful reproduction may therefore require a male to succeed at several stages (Andersson 1994; Whitlock \& Agrawal 2009). First, he must search for and locate (or attract) a female, potentially competing indirectly with other males in his ability to do this (i.e. scramble competition). He may then have to compete directly for access to her (i.e. contest competition among males), as well as court or otherwise coerce her into mating. Finally, after mating he may have to guard her against rival suitors or have his sperm compete with that of other males.

Electronic supplementary material is available at http://dx.doi.org 10.1098/rsbl.2009.0475 or via http://rsbl.royalsocietypublishing.org.
Although Darwin's (1859) original description of sexual selection in the Origin of Species recognized that it may often act in concert with natural selection, favouring the most vigorous and best adapted males, his focus quickly narrowed to that subset of characters whose evolution could not be explained by natural selection alone. Darwin (1871) paid particular attention to the evolution of the elaborate sexual display traits and weaponry found in the males of many species. Since then, female preferences and malemale competition have been the components of sexual selection that have received the most attention (Andersson 1994; Whitlock \& Agrawal 2009). Although consideration has been given to the effects on trait evolution of other components of sexual fitness, such as sperm competition (e.g. Harcourt et al. 1981) and search effort (e.g. Able 1999; Foellmer \& Fairbairn 2005; Kelly et al. 2008), the population genetic consequences of these components in terms of population mean fitness remain largely overlooked.

It has been suggested by several authors that, if mutations harmful to population mean fitness also tend to reduce male reproductive success, then sexual selection may have an important consequence by reducing the mutation load (Whitlock 2000; Siller 2001; Agrawal 2001; reviewed in Whitlock \& Agrawal 2009). Provided that females mate with other males, deleterious mutations may be purged from a population via sexual selection on males without a corresponding reduction in population mean fitness. Evaluating the consequences of sexual selection on the frequency of deleterious mutations requires determining whether total selection against such mutations is stronger in males than in females (Whitlock \& Agrawal 2009). Here we are interested in a potentially important contributor to variance in male reproductive success that has been overlooked in this regard: searching for and locating a mate. Failure to locate a female precludes the possibility of any reproductive success, so by strengthening sexual selection on males, search effort may have important population genetic consequences in terms of purging deleterious mutations.

Males often invest substantial time and energy in searching for potential mates and such investment is costly (Kokko \& Wong 2007). Search effort is therefore expected to be condition-dependent: males of lower quality will have fewer resources overall and allocation to search effort is likely to suffer (Whitlock \& Agrawal 2009). Because most genes in the genome are likely to contribute to an individual's overall condition (Rowe \& Houle 1996), variable search effort is likely to reinforce natural selection, favouring the same alleles responsible for high condition and hence fitness.

Here we quantify the contribution of search effort to sexual selection against 10 independent, presumed deleterious, mutations with visible phenotypic effects in a laboratory population of Drosophila melanogaster. Specifically, we ask whether including a component of search effort lowers the reproductive success of mutant relative to wild-type males in replicate binomial mating trials, as would be expected if search effort is an important component of sexual selection against these mutations. We directly manipulate the opportunity for search effort by conducting the mating trials in two different sized arenas that vary approximately 600fold in volume. If search effort is an important 
condition-dependent component of male sexual fitness, then mutant males should suffer disproportionately relative to wild-type males in the larger arena in which it is more difficult and/or more costly for them to locate the female.

\section{MATERIAL AND METHODS}

\section{(a) Study populations}

Ten mutant lines were obtained from the Bloomington stock centre. Three of these, yellow $(y)$, forked $(f)$ and white $(w)$, were fixed for different X-linked visible recessive mutations, and seven of them, brown (bw), claret (ca), cinnabar (cn), eyeless (ey), plexus $(p x)$, sepia $(s e)$ and speck $(s p)$, were fixed for different autosomal visible recessive mutations. Six of these mutations affect eye phenotype (bw, w, cn, ca, se, ey), two affect wing phenotype (sp and $p x$ ), and one each affect bristles $(f)$ and body colour $(y)$. Each mutation was introgressed separately into a stock population (see the electronic supplementary material for a description of this population) via five rounds of backcrossing, thereby yielding 10 populations that shared a similar wild-type stock genetic background, but was each fixed for a different mutation. At least one round of backcrossing was initiated by a cross in each direction (i.e. stock female $\times$ mutant male and mutant female $\times$ stock male) to ensure that the final introgressed mutant populations carried mtDNA and Y chromosomes from the stock. After backcrossing, mutant populations were maintained under the same conditions as the stock.

\section{(b) Mating assays}

Flies were collected as virgins upon emergence using light $\mathrm{CO}_{2}$ anaesthesia and held separately by sex in vials with $5 \mathrm{ml}$ of standard medium for 4-5 days prior to their use in the mating trials. For each population, 98-100 replicate binomial mating trials were performed simultaneously under each of two experimental treatments that differed in arena size. Each trial consisted of a single mutant female from one of the populations together with a mutant male from the same population and a stock (wild-type) male. In the small mating arenas, trials were conducted in standard glass Drosophila rearing vials of approximately $30 \mathrm{ml}$ volume with $10 \mathrm{ml}$ of standard medium, whereas in the large mating arena, trials were conducted in rectangular cages of approximately 191 in volume (for a detailed description, see the electronic supplementary material). Individuals remained in both mating treatments for $24 \mathrm{~h}$, after which the males were discarded and females were removed, individually transferred to separate vials containing $10 \mathrm{ml}$ standard food for $24 \mathrm{~h}$ of egglaying, and finally discarded. All adult offspring produced by each female were subsequently counted and scored by phenotype (mutant versus wild-type).

\section{(c) Statistical analyses}

The reproductive success of mutant relative to stock males was calculated for each mutation as a proportion $(p)$ by dividing the total number of mutant offspring produced by all females of that mutation by the total number of offspring produced by the same females (mutant + wild-type). Calculating relative mating success in this way weighs each female by her total fecundity, as would occur in nature.

Because we are primarily interested in the contribution of search effort to male sexual fitness in general, we conducted a single overall test of whether sexual selection against these mutations was stronger in the large when compared with the small arena. A one-sample $t$-test, treating the 10 mutations as replicates, was used to determine whether the difference in mean proportion differed from zero (i.e. $\bar{p}_{\text {vial }}-\bar{p}_{\text {cage }}=0$; sexual fitness of mutant relative to stock males is the same in the small versus large arena). To test for heterogeneity among mutations in the effect of the mating arena treatment, individual $p$-values were calculated for every female. These values were bimodally distributed, however, because most females produced a majority of offspring of one type or the other (i.e. mutant or wild-type). We therefore assigned a binomial score to each replicate female in which a value of ' 1 ' was given to all females for which $p \geq 0.5$ and a value of ' 0 ' when $p<0.5$ (results do not depend on the assignment of females for which $p \equiv 0.5$ ). To test for heterogeneity among mutations in the effect of the mating arenas, these binomial score data were modelled as a linear function of the fixed effects of treatment (vial versus cage), mutation and their interaction. The model was fit using maximum likelihood and a logistic link function, with significance of the terms determined using likelihood ratio tests. The effect of search effort on each

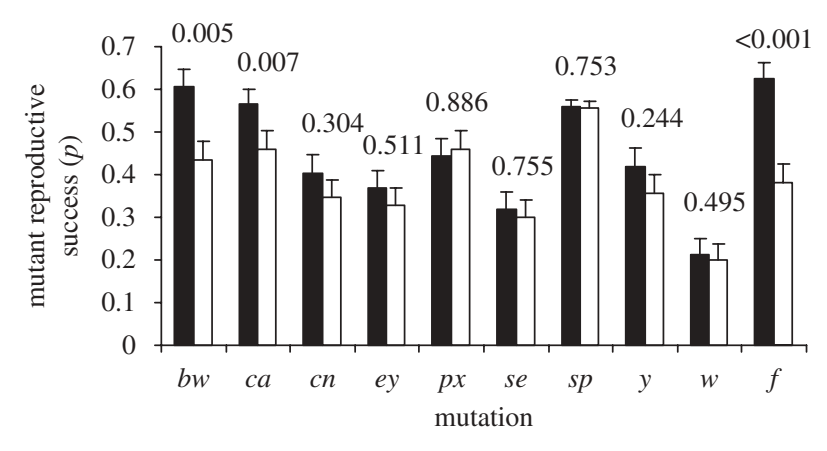

Figure 1. Reproductive success of mutant relative to stock males (proportion of mutant offspring, $p \pm$ s.e.) for 100 replicate mating trials conducted in small (black bars) and large (white bars) mating arenas for each of 10 mutations. $p$-values across the top are from separate tests comparing small versus large arenas, treating individuals as replicates.

individual mutation was tested using a simplified version of this model that was fit separately for each mutation after removal of the mutant and mutant $\times$ treatment terms.

\section{RESULTS}

Average reproductive success of mutant relative to stock males, as measured by the proportion of mutant offspring $(p)$ produced by females, was lower in the large cages when compared with the small vials for nine of the 10 mutations (figure 1). This difference is significant overall $\left(t_{9}=2.70, p=0.025\right)$ and represents a $6.6 \pm 2.4$ per cent (mean \pm s.e.) reduction in the proportion of mutant offspring in cages than in vials. Significant heterogeneity was detected among the mutations in the effect of the mating arena $\left(\chi^{2}=17.03, \quad\right.$ d.f. $\left.=9, \quad p=0.048\right)$. Within the individual mutations, the effect of mating arena was significant in three cases, in all of which mutant males suffered disproportionately in the large when compared with the small arenas (figure 1).

\section{DISCUSSION}

For males of many species, the initial step in reproducing is to successfully locate a female. Although not previously considered in this respect, here we have shown that sexual selection against deleterious mutations in males tends to be stronger overall when an opportunity for search effort is included than when it is excluded. Significant heterogeneity existed among mutations in the effects of search effort. Such heterogeneity is expected because, along with their indirect effects on sexual fitness mediated via reductions in male health or condition, individual mutations may have separate direct effects on sexual fitness. Such effects may be particularly likely given that the mutations we used had large and visible effects that may impinge on a male's search ability (e.g. eye and wing mutations). An important goal of future research will be to determine the generality of these results with respect to mutations of small effects segregating within natural populations.

Cages reduced female remating rates when compared with vials, as indicated by a greater number of individual females producing both mutant and wild-type offspring in the latter when compared 
with the former (results not shown). Differential sperm competition may therefore have existed between our treatments, although to explain our results mutant males would have to consistently outperform wild-type males in this respect. Preliminary data suggest the opposite, however, with stock males outperforming mutant males in sperm offensive ability for four of five mutations tested (J. Sztepanacz 2009, unpublished results).

Variable search effort may be of particular importance because it provides a potential mechanism by which sexual selection can reinforce natural selection that does not rely on the evolution or maintenance of costly female preferences as required by a good genes process. In this scenario, females mate with higher condition males not because of any innate preference for exaggerated displays exhibited by these males, but because low condition males are simply not present, or they arrive too late.

In species where population mean fitness is determined by the number and fecundity of females, mutation load is reduced whenever total selection against deleterious mutations is stronger in males than in females (Whitlock \& Agrawal 2009). By strengthening sexual selection on males, search effort is potentially an important component of sexual fitness that needs to be considered. During mate search, males may experience an increase in various risks (e.g. predation) or heightened exposure to certain stresses (e.g. starvation, desiccation) relative to females. Although blurring the distinction between natural and sexual selection, such processes may further aid in reducing mutation load. How search effort covaries with other components of sexual and non-sexual fitness is an important topic for future research.

We thank S. Khair, V. Rontondo and P. Wu for help in the laboratory. R. Dukas kindly provided the stock population of $D$. melanogaster. Two anonymous reviewers provided useful comments on a previous version. This work was funded by a grant to H.D.R. from the Natural Sciences and Engineering Research Council of Canada and an Ontario Early Researcher Award.
Able, D. J. 1999 Scramble competition selects for greater tailfin size in male red-spotted newts (Amphibia: Salamandridae). Behav. Ecol. Sociobiol. 46, 423-428. (doi:10.1007/s002650050638)

Agrawal, A. F. 2001 Sexual selection and the maintenance of sexual reproduction. Nature 411, 692-695. (doi:10.1038/ 35079590)

Andersson, M. 1994 Sexual selection. Princeton, NJ: Princeton University Press.

Darwin, C. 1859 The origin of species by means of natural selection. London, UK: Murray.

Darwin, C. 1871 The descent of man, and selection in relation to sex. London, UK: Murray.

Foellmer, M. W. \& Fairbairn, D. J. 2005 Selection on male size, leg length and condition during mate search in a sexually highly dimorphic orb-weaving spider. Oecologia 142, 653-662. (doi:10.1007/s00442-0041756-3)

Harcourt, A. H., Harvey, P. H., Larson, S. G. \& Short, R. V. 1981 Testis weight, body weight and breeding system in primates. Nature 293, 55-67. (doi:10.1038/ 293055a0)

Kelly, C. D., Bussière, L. F. \& Gwynne, D. T. 2008 Sexual selection for male mobility in a giant insect with femalebiased size dimorphism. Am. Nat. 172, 417-423. (doi:10.1086/589894)

Kokko, H. \& Wong, B. B. M. 2007 What determines sex roles in mate searching? Evolution 61, 1162-1175. (doi:10.1111/j.1558-5646.2007.00090.x)

Murphy, C. G. 1998 Interaction-independent sexual selection and the mechanisms of sexual selection. Evolution 52, 8-18. (doi:10.2307/2410915)

Rowe, L. \& Houle, D. 1996 The lek paradox and the capture of genetic variance by condition dependent traits. Proc. R. Soc. Lond. B 263, 1415-1421. (doi:10.1098/ rspb.1996.0207)

Siller, S. 2001 Sexual selection and the maintenance of sex. Nature 411, 689-692. (doi:10.1038/35079578)

Whitlock, M. C. 2000 Fixation of new alleles and the extinction of small populations: drift load, beneficial alleles, and sexual selection. Evolution 54, 1855-1861.

Whitlock, M. C. \& Agrawal, A. F. 2009 Purging the genome with sexual selection: reducing mutation load through selection on males. Evolution 63, 569-582. (doi:10. $1111 / \mathrm{j} .1558-5646.2008 .00558 . x)$ 